\title{
A!
}

This is an electronic reprint of the original article.

This reprint may differ from the original in pagination and typographic detail.

López González, Diego; Casiraghi, Arianna; Van De Wiele, Ben; Van Dijken, Sebastiaan Reconfigurable magnetic logic based on the energetics of pinned domain walls

Published in:

Applied Physics Letters

DOI:

$10.1063 / 1.4940119$

Published: 18/01/2016

Document Version

Publisher's PDF, also known as Version of record

Please cite the original version:

López González, D., Casiraghi, A., Van De Wiele, B., \& Van Dijken, S. (2016). Reconfigurable magnetic logic based on the energetics of pinned domain walls. Applied Physics Letters, 108(3), 1-4. [032402].

https://doi.org/10.1063/1.4940119

This material is protected by copyright and other intellectual property rights, and duplication or sale of all or part of any of the repository collections is not permitted, except that material may be duplicated by you for your research use or educational purposes in electronic or print form. You must obtain permission for any other use. Electronic or print copies may not be offered, whether for sale or otherwise to anyone who is not an authorised user. 


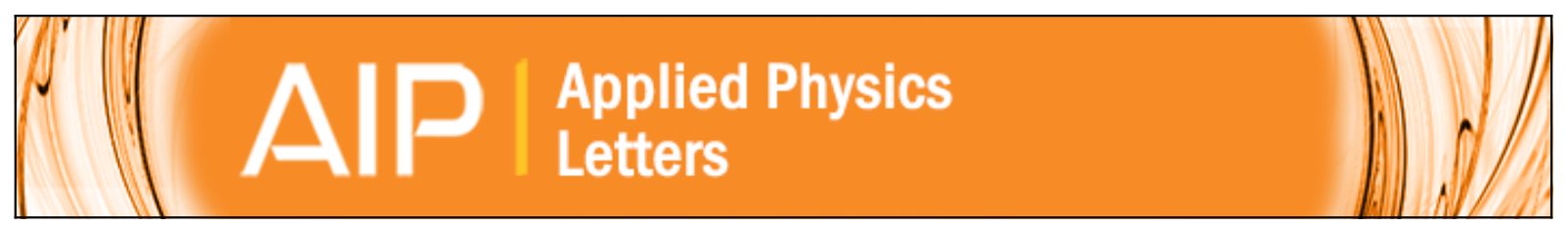

\section{Reconfigurable magnetic logic based on the energetics of pinned domain walls}

Diego López González, Arianna Casiraghi, Ben Van de Wiele, and Sebastiaan van Dijken

Citation: Applied Physics Letters 108, 032402 (2016); doi: 10.1063/1.4940119

View online: http://dx.doi.org/10.1063/1.4940119

View Table of Contents: http://scitation.aip.org/content/aip/journal/apl/108/3?ver=pdfcov

Published by the AIP Publishing

\section{Articles you may be interested in}

Stability of a pinned magnetic domain wall as a function of its internal configuration

J. Appl. Phys. 117, 023909 (2015); 10.1063/1.4905245

Controlled domain wall pinning in nanowires with perpendicular magnetic anisotropy by localized fringing fields J. Appl. Phys. 115, 17D506 (2014); 10.1063/1.4864737

Realization of a mesoscopic reprogrammable magnetic logic based on a nanoscale reconfigurable magnonic crystal

Appl. Phys. Lett. 100, 073114 (2012); 10.1063/1.3687177

Domain wall assisted magnetization switching in (111) oriented L 10 FePt grown on a soft magnetic metallic glass

Appl. Phys. Lett. 97, 072510 (2010); 10.1063/1.3479054

Anomalous switching in submicrometer magnetic tunnel junction arrays arising from magnetic vortex and domain wall pinning

J. Appl. Phys. 96, 1748 (2004); 10.1063/1.1765852

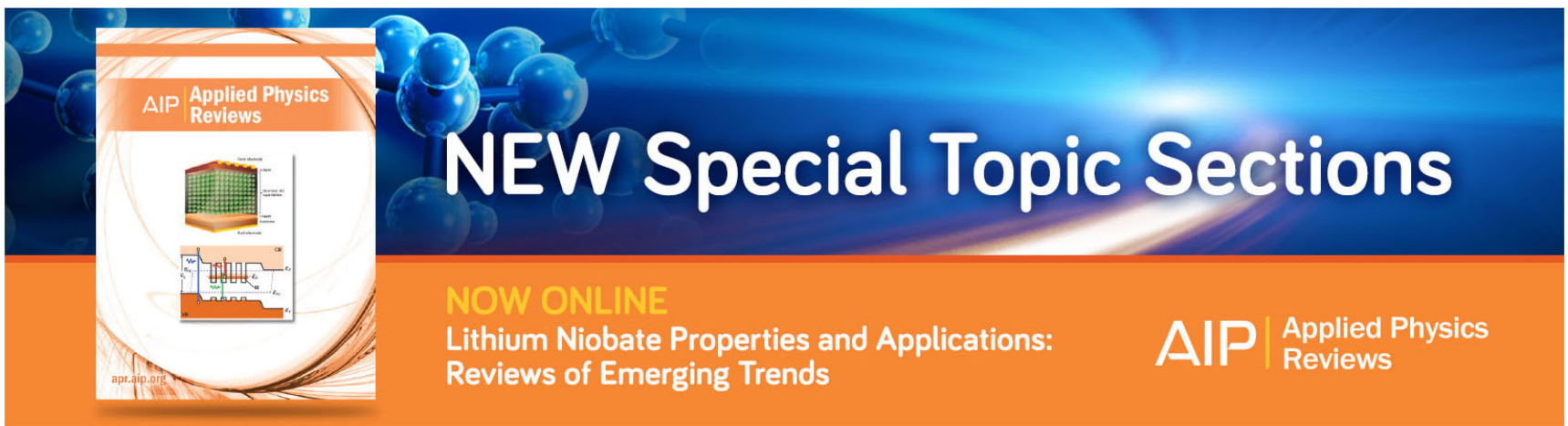




\title{
Reconfigurable magnetic logic based on the energetics of pinned domain walls
}

\author{
Diego López González, ${ }^{1}$ Arianna Casiraghi, ${ }^{1}$ Ben Van de Wiele, ${ }^{2}$ \\ and Sebastiaan van Dijken ${ }^{1, a)}$ \\ ${ }^{1}$ NanoSpin, Department of Applied Physics, Aalto University School of Science, P.O. Box 15100, \\ FI-00076 Aalto, Finland \\ ${ }^{2}$ Department of Electrical Energy, Systems and Automation, Ghent University, Ghent B-9000, Belgium
}

(Received 13 October 2015; accepted 6 January 2016; published online 19 January 2016)

\begin{abstract}
A magnetic logic concept based on magnetic switching in three stripe domains separated by pinned magnetic domain walls is proposed. The relation between the inputs and the output of the logic operator is determined by the energetics of the domain walls, which can be switched between two distinctive states by an external magnetic field. Together with magnetic read-out along two orthogonal directions, non-volatile AND, OR, NAND, and NOR gates can be created. The logic concept is experimentally demonstrated using $\mathrm{CoFeB}$ films on $\mathrm{BaTiO}_{3}$ substrates, and micromagnetic simulations are used to analyze the energetics of the system. (C) 2016 AIP Publishing LLC.
\end{abstract}

[http://dx.doi.org/10.1063/1.4940119]

The use of magnetic elements in logic devices has attracted attention as an alternative to semiconductor-based technologies. Advantages of magnetic logic include low energy consumption, non-volatility, high processing rates, and reprogrammability. Several magnetic structures and interaction mechanisms have been proposed. Among them are concepts based on magnetic switching in magnetoresistive elements, ${ }^{1}$ magnetic domain wall motion in nanowires, ${ }^{2}$ magnetostatic interactions between nanomagnets, ${ }^{3-7}$ spin transport in coherent channels, ${ }^{8}$ magnetic exchange coupling between single atoms, ${ }^{9}$ and interlayer exchange coupling in threedimensional magnetic ratchets. ${ }^{10}$ Magnonic logic based on the manipulation of spin waves has also been studied. ${ }^{11-13}$

In this letter, we report on a different type of non-volatile and reconfigurable magnetic logic. The concept is based on the functionalization of pinned magnetic domain walls, which requires regular modulations of magnetic anisotropy with abrupt and straight anisotropy boundaries. For the proof-of-concept experiments presented here, strain coupling between a $100 \mathrm{~nm}$ thick $\mathrm{Co}_{40} \mathrm{Fe}_{40} \mathrm{~B}_{20}$ film and a ferroelectric $\mathrm{BaTiO}_{3}$ substrate with ferroelastic $a_{1}-a_{2}$ stripe domains is used to induce magnetic anisotropy modulations. ${ }^{14-17}$ In this system, inverse magnetostriction produces a strong correlation between the direction of magnetic anisotropy in the CoFeB film and the lattice tetragonality (and orientation of the ferroelectric polarization) in the $\mathrm{BaTiO}_{3}$ domains. For the selected sample, the magnetic anisotropy is uniaxial and the easy axis of magnetization rotates abruptly by $90^{\circ}$ at domain boundaries. As a consequence, strong pinning of magnetic domain walls on top of perfectly straight ferroelectric domain boundaries is attained. ${ }^{14,15,18}$ The magnetic configuration of the hybrid system and its use as a magnetic logic element is schematically illustrated in Fig. 1.

Key to the logic concept is the ability to switch between energetically different magnetic domain walls at fixed pinning positions. ${ }^{19}$ If a magnetic field is applied along the

a) sebastiaan.van.dijken@aalto.fi stripe domains of Fig. 1, alternating head-to-head and tail-totail domain walls are formed. The magnetic energy of these domain walls is high (HE walls). On the other hand, if the magnetic field is applied perpendicular to the stripe domains, low-energy (LE) head-to-tail domain walls are created. The difference in magnetic energy between HE and LE domain walls has a pronounced influence on the magnetization reversal process in the $\mathrm{CoFeB}$ film. ${ }^{20}$ This is illustrated by the magneto-optical Kerr effect (MOKE) microscopy images of Fig. 2. Here, a saturating magnetic field is first applied parallel to the stripe domains. When the field is reduced, pinned HE domain walls form and their spin rotation, width, and energy gradually increase. This process continues upon field reversal until the magnetization of every second stripe domain switches at 12-13 mT. After these switching events, all domains are separated by LE domain walls. The new magnetic configuration is stable up to a field of $24 \mathrm{mT}$. A further increase of the magnetic field results in abrupt magnetic switching in the remaining stripe domains and the formation of small-angle HE domain walls with modest energy. The strong lateral modulation of magnetization reversal in the $\mathrm{CoFeB}$ film is thus driven by energy minimization via $\mathrm{HE}$ to LE domain wall transformations.

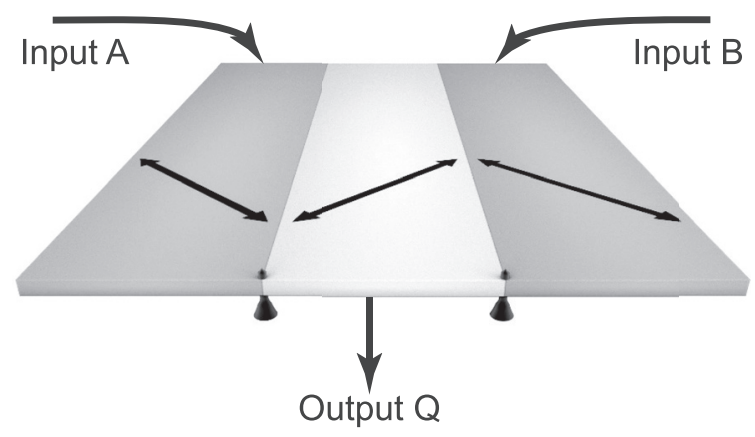

FIG. 1. Illustration of the logic concept. The element consists of three magnetic stripe domains with abrupt $90^{\circ}$ rotations of the uniaxial magnetic anisotropy (double-headed arrows) and two pinned magnetic domain walls. The outer domains are used as inputs and the central domain functions as output. 


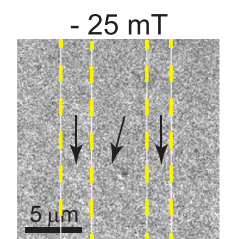
$13 \mathrm{mT}$
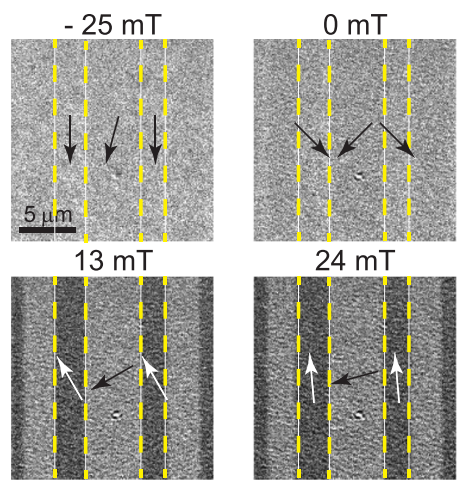
$24 \mathrm{mT}$
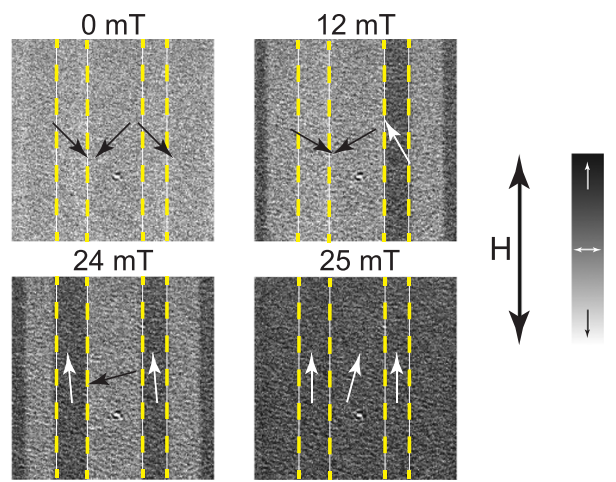

FIG. 2. MOKE microscopy images illustrating magnetization reversal in a $100 \mathrm{~nm}$ thick $\mathrm{CoFeB}$ film on $\mathrm{BaTiO}_{3}$. The magnetic field is applied parallel to the stripe domains and the arrows indicate the direction of magnetization.

The reversal process in the $\mathrm{CoFeB}$ film allows for the initialization of four magnetic configurations when three stripe domains with different width are considered (images in the top row of Fig. 3(a)). The four magnetization states correspond to the presence of two HE, one LE and one HE (or vice versa), and two LE domain walls. Because of dissimilar energies, the central domain switches at distinctive magnetic fields for each of these configurations. Experimental switching curves for a magnetic field opposing the remanent magnetization of the central domain are shown in Fig. 3(b). The central domain switches first for the HE-HE state. In this case, the switching event is driven by energy minimization via two HE-to-LE wall transformations, favoring early switching at a reduced magnetic field. The central domains of the LE-HE and HE-LE configurations switch next. Both before and after these switching events, the system consists of one $\mathrm{HE}$ and one LE domain wall. Magnetic switching is therefore hardly affected by the energetics of the domain walls. Finally, magnetization reversal in the central domain of the LE-LE state involves the formation of two energetically unfavorable HE domain walls and, hence, requires a larger magnetic field. Importantly, magnetic switching in the central domain does not affect the magnetization state of the outer domains.

Exploiting the circumstance that each configuration exhibits a characteristic switching field for the central domain, we now propose a magnetic logic element with reconfigurable functions. To this end, we identify the two outer

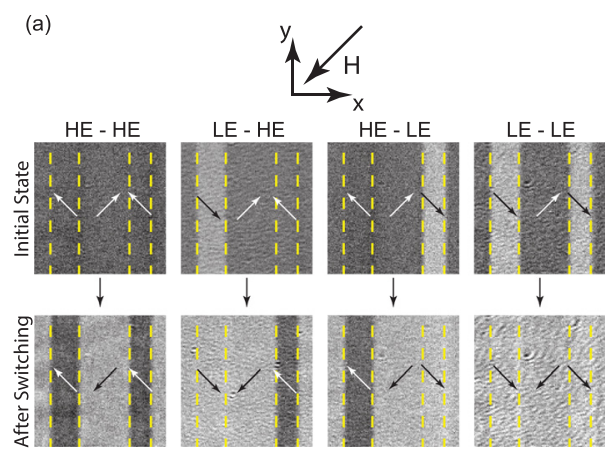

(a)

(1) $\left(9 \mathrm{mT}<\mu_{0} \mathrm{H}<15 \mathrm{mT}\right)$
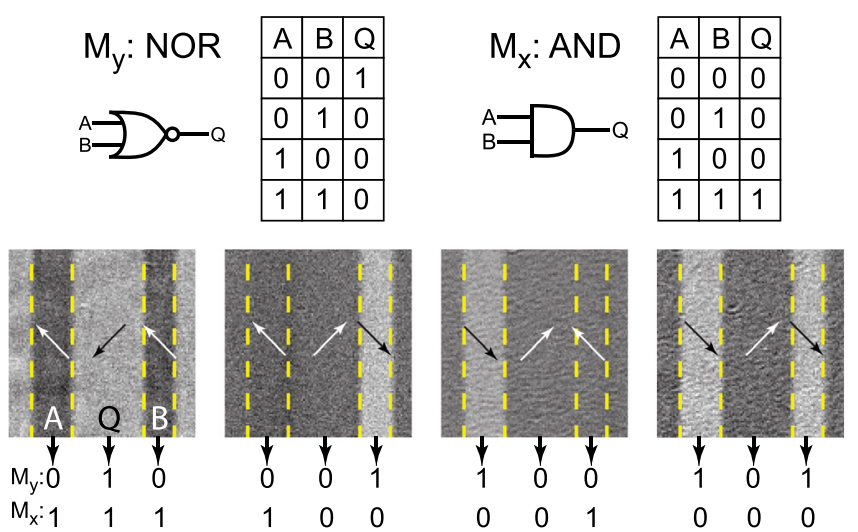

(b)

(2) $\left(15 \mathrm{mT}<\mu_{0} \mathrm{H}<18 \mathrm{mT}\right)$
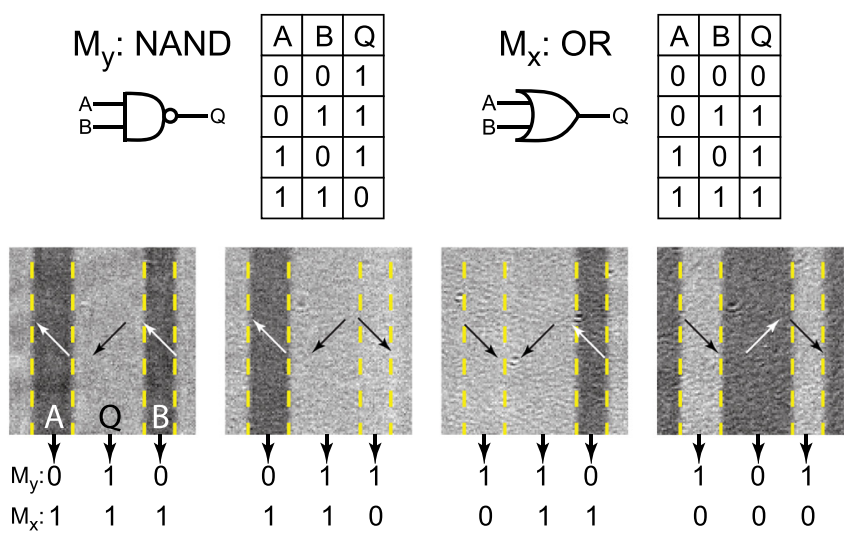

FIG. 4. Demonstration of reconfigurable magnetic logic. For 9 $\mathrm{mT}<\mu_{0} H<15 \mathrm{mT}$ (a), the element functions as a NOR or an AND gate depending on the magnetization component that is read out. Operations corresponding to a NAND or an OR gate are performed for $15 \mathrm{mT}<\mu_{0} H<18 \mathrm{mT}$ (b). The MOKE microscopy images in (a) and (b) represent the remanent state of the HE-HE (left), LE-HE (second from left), HE-LE (third from left), and LE-LE (right) configurations after application of a magnetic field in one of the two working regimes. Corresponding truth tables are extracted from the measurements by using labels " 0 " and " 1 " for magnetization pointing up and down $\left(M_{y}\right)$ or magnetization pointing right and left $\left(M_{x}\right)$, respectively.

domains as inputs (A and B) and the central domain as output $(\mathrm{Q})$. Furthermore, we first consider the magnetization component along the $\mathrm{y}$-axis $\left(M_{y}\right)$ pointing up (dark MOKE contrast) as logic state "0" and pointing down (bright MOKE

(b)

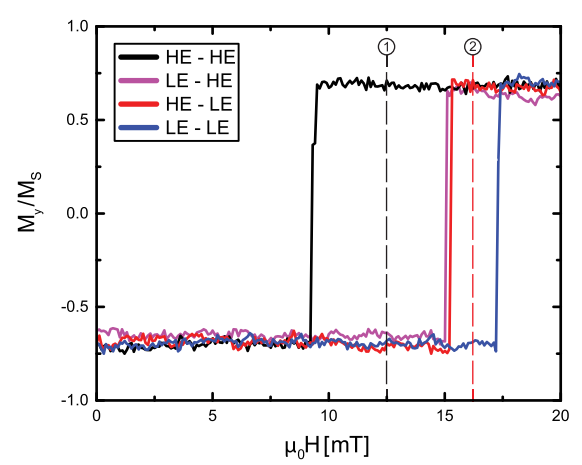

FIG. 3. (a) MOKE microscopy images of the remanent HE-HE, LE-HE, HE-LE, and LE-LE configurations at initialization and after switching in the central domain. The arrows indicate the direction of magnetization, and the dashed yellow lines mark the location of pinned domain walls. (b) Magnetic switching curves for the central domain. The normalized magnetization along the $y$-axis is plotted as a function of magnetic field. The field direction is indicated by the arrow above the images in (a). Symbols (1) and (2) mark magnetic fields that correspond to different working regimes of the logic element (see Fig. 4). 
contrast) as logic state "1." If a magnetic field is applied between the switching fields of the HE-HE and LE-HE/ HE-LE configurations (marked by (1) in Fig. 3(b)), the magnetic output switches from " 0 " to " 1 " only if both inputs are off (left image in Fig. 4(a)). In the other three cases, the output signal remains " 0. ." The truth table of this logic element corresponds to a NOR gate. If the magnetic field is increased beyond the switching field of the LE-HE and HE-LE configurations, but below that of the LE-LE state (marked by (2) in Fig. 3(b)), the magnetic output turns on if at least one of the two inputs is off (three left images in Fig. 4(b)). The logic element now functions as a NAND gate. A similar sequence of switching events can be described when the magnetization component along the $\mathrm{x}$-axis $\left(M_{x}\right)$ is used. In this case, the logic element operates as an AND or an OR gate, depending on bias field. Measurements of $M_{x}$ and $M_{y}$ can, for example, be performed by setting the reference layer of a magnetoresistive element on top of the magnetic output. In that case, the logic functionality (AND/OR or NAND/NOR) has to be predefined before the logic operation is executed. We note that a NOT gate can easily be constructed by considering two magnetic stripe domains separated by a LE domain wall (not shown). Reversal of the magnetic field resets the system for new operations.

To further analyze the energetics of the reconfigurable logic element, we performed micromagnetic simulations using MuMax $3 .^{21}$ In agreement with the experiments, a uniaxial magnetic anisotropy of $K_{u}=3 \times 10^{4} \mathrm{~J} / \mathrm{m}^{3}$ and stripe domain widths of $2.5 \mu \mathrm{m}$ (left), $4.6 \mu \mathrm{m}$ (center), and $2.1 \mu \mathrm{m}$ (right) were used. Figure 5(a) summarizes the contributions of the anisotropy energy $\left(E_{a n}\right)$, magnetostatic energy $\left(E_{m s}\right)$, and exchange energy $\left(E_{e x}\right)$ to the total energy density $\left(E_{t}\right)$ of two remanent states, one with two LE and the other with two HE domain walls. For the LE-LE configuration, the domain walls are narrow and the magnetic energies are enhanced only near the anisotropy boundaries. The broader domain walls in the HE-HE state result in large $E_{a n}$ and $E_{m s}$ over the entire width of the stripe domains. Averaged over the three domains, the total energy density $E_{t}$ of the remanent LE-LE configuration is about $10^{4} \mathrm{~J} / \mathrm{m}^{3}$ smaller than that of the HE-HE state.

The application of a magnetic field gradually changes the energy landscape. Figure 5(b) shows simulation results for $E_{t}$ and a magnetic field opposing the remanent magnetization in the central domain (i.e., the configuration is identical to the experiments of Fig. 3). In addition to the LE-LE and HE-HE cases, the LE-HE and HE-LE configurations are also included. Initially, $E_{t}$ increases for all magnetic configurations due to an increase of the spin rotation within the domain walls and a growing misalignment between the magnetization and anisotropy axes inside the outer domains. Abrupt switching of the magnetization in the central domain lowers $E_{t}$ of the HE-HE state first. This early switching event is triggered by two HE-to-LE domain wall transformations. The decrease of $E_{t}$ in this case is caused by a switch of the magnetic domain wall type and a lowering of the Zeeman energy. Abrupt magnetization reversal in the central domain of the LE-HE and HE-LE configurations requires a larger magnetic field, since it is less affected by the energetics of the domain walls. Finally, magnetic switching in the LE-LE state is postponed because it involves two energetically unfavorable LE-to-HE domain wall transformations. The switching curves of Fig. 5(c) are in a good agreement with the experimental results of Fig. 3.

Scalability of the magnetic logic concept is limited by finite-size effects of magnetic domain formation. Magnetic anisotropy modulations give rise to magnetic stripe domains if the width of the domain walls is comparable or smaller than the width of the modulation. ${ }^{19}$ Since the logic operator is based on the functionalization of two types of domain walls, the broader HE wall is the limiting factor. In the experiments presented here, the HE domain walls are a few micrometers wide. Downscaling of the concept can be achieved by an increase of the anisotropy strength or a reduction of the magnetic film thickness. For example, for $K_{u}=3 \times 10^{5} \mathrm{~J} / \mathrm{m}^{3}$ and $t_{F M}=50 \mathrm{~nm}$, domain sizes of about $100 \mathrm{~nm}$ are sufficient to implement logic operations. Simulations using the same parameters indicate that downscaling in the other direction (i.e., parallel to the domain walls) to $20 \mathrm{~nm}$ still results in separate magnetic switching events for different combinations of LE and HE domain walls.

In summary, we have demonstrated a magnetic logic concept based on three magnetic stripe domains and two pinned magnetic domain walls. The concept requires a lateral modulation of magnetic anisotropy with abrupt boundaries and (a)

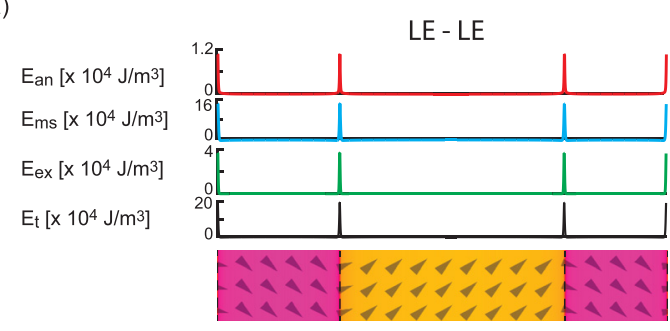

HE - HE

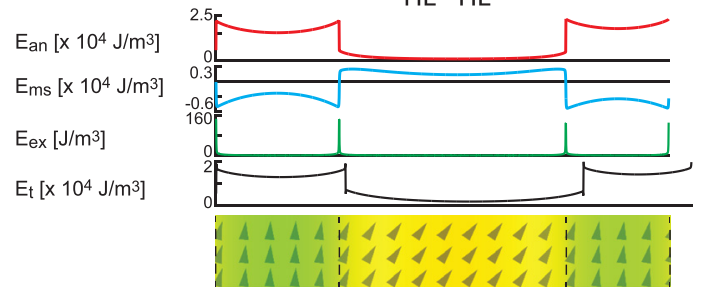

(b)

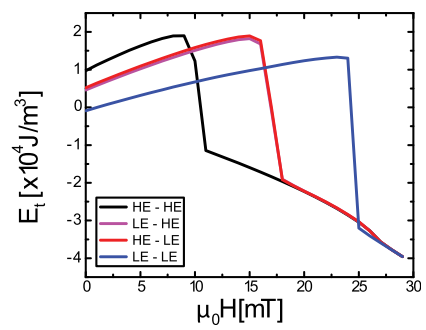

(c)

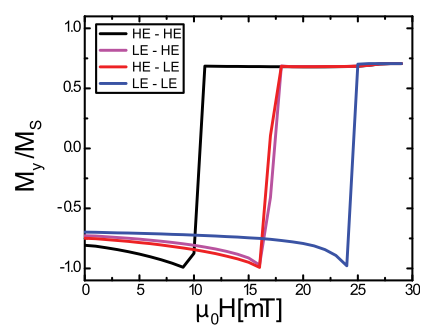

FIG. 5. (a) Micromagnetic simulations of the remanent LE-LE and HE-HE magnetization states and plots of the corresponding energy densities. (b) Total energy density as a function of applied magnetic field for all four domain configurations. (c) Simulated magnetic switching curves for $M_{y}$ in the central domain. 
initialization of two types of pinned magnetic domain walls with distinctive energies. Experiments on strain-coupled domain patterns in $\mathrm{CoFeB} / \mathrm{BaTiO}_{3}$ indicate that different logic operations (AND, OR, NAND, and NOR) can be performed with the same logic element.

This work was supported by the Academy of Finland (Grant No. 260361) and the European Research Council (Nos. ERC-2012-StG 307502-E-CONTROL and ERC-2014PoC 665215-EMOTION).

${ }^{1}$ A. Ney, C. Pampuch, R. Koch, and K. Ploog, Nature 425, 485 (2003).

${ }^{2}$ D. A. Allwood, G. Xiong, C. Faulkner, D. Atkinson, D. Petit, and R. Cowburn, Science 309, 1688 (2005).

${ }^{3}$ R. Cowburn and M. Welland, Science 287, 1466 (2000).

${ }^{4}$ A. Imre, G. Csaba, L. Ji, A. Orlov, G. Bernstein, and W. Porod, Science 311, 205 (2006).

${ }^{5}$ D. B. Carlton, N. C. Emley, E. Tuchfeld, and J. Bokor, Nano Lett. 8, 4173 (2008).

${ }^{6}$ D. Bhowmik, L. You, and S. Salahuddin, Nat. Nanotechnol. 9, 59 (2014).

${ }^{7}$ Z. Gu, M. E. Nowakowski, D. B. Carlton, R. Storz, M.-Y. Im, J. Hong, W. Chao, B. Lambson, P. Bennett, M. T. Alam et al., Nat. Commun. 6, 6466 (2015).

${ }^{8}$ B. Behin-Aein, D. Datta, S. Salahuddin, and S. Datta, Nat. Nanotechnol. 5, 266 (2010).
${ }^{9}$ A. A. Khajetoorians, J. Wiebe, B. Chilian, and R. Wiesendanger, Science 332, 1062 (2011).

${ }^{10}$ R. Lavrijsen, J.-H. Lee, A. Fernández-Pacheco, D. C. Petit, R. Mansell, and R. P. Cowburn, Nature 493, 647 (2013).

${ }^{11}$ T. Schneider, A. Serga, B. Leven, B. Hillebrands, R. Stamps, and M. Kostylev, Appl. Phys. Lett. 92, 022505 (2008).

${ }^{12}$ A. V. Chumak, A. A. Serga, and B. Hillebrands, Nat. Commun. 5, 4700 (2014).

${ }^{13}$ A. V. Chumak, V. I. Vasyuchka, A. A. Serga, and B. Hillebrands, Nat. Phys. 11, 453 (2015).

${ }^{14}$ T. H. E. Lahtinen, J. O. Tuomi, and S. van Dijken, Adv. Mater. 23, 3187 (2011).

${ }^{15}$ T. H. Lahtinen, K. J. Franke, and S. van Dijken, Sci. Rep. 2, 258 (2012).

${ }^{16}$ R. Chopdekar, V. K. Malik, A. F. Rodríguez, L. Le Guyader, Y. Takamura, A. Scholl, D. Stender, C. Schneider, C. Bernhard, F. Nolting et al., Phys. Rev. B 86, 014408 (2012).

${ }^{17}$ R. Streubel, D. Köhler, R. Schäfer, and L. M. Eng, Phys. Rev. B 87, 054410 (2013).

${ }^{18}$ K. J. Franke, T. H. Lahtinen, and S. van Dijken, Phys. Rev. B 85, 094423 (2012).

${ }^{19}$ K. J. Franke, D. López González, S. J. Hämäläinen, and S. van Dijken, Phys. Rev. Lett. 112, 017201 (2014).

${ }^{20}$ A. Casiraghi, T. Rincón Domínguez, S. Rößler, K. J. A. Franke, D. López González, S. J. Hämäläinen, R. Frömter, H. P. Oepen, and S. van Dijken, Phys. Rev. B 92, 054406 (2015)

${ }^{21}$ A. Vansteenkiste, J. Leliaert, M. Dvornik, M. Helsen, F. Garcia-Sanchez, and B. Van Waeyenberge, AIP Adv. 4, 107133 (2014). 